\begin{tabular}{|c|l|}
\hline Title & Extremely early stage of ferroelastic domain formation observed by laser refraction \\
\hline Author(s) & Kinoshita, S.; Kutsuzawa, S.; Shimada, Y.; Y agi, T. \\
\hline Citation & $\begin{array}{l}\text { Physical Review B, 50(9), 5834 5837 } \\
\text { https://doi.org/10.1103/PhysRevB.50.5834 }\end{array}$ \\
\hline Issue Date & 199409-01 \\
\hline Doc URL & http://hdl.handle.net/2115/6111 \\
\hline Rights & Copyright $\odot$ 1994 A merican Physical Society \\
\hline Type & article \\
\hline File Information & PRB50.pdf \\
\hline
\end{tabular}

Instructions for use 


\title{
Extremely early stage of ferroelastic domain formation observed by laser refraction
}

\author{
S. Kinoshita, ${ }^{*}$ S. Kutsuzawa, Y. Shimada, and T. Yagi \\ Research Institute for Electronic Science, Hokkaido University, Sapporo 060, Japan
}

(Received 6 June 1994)

The initial domain formation process in a ferroelastic crystal $\mathrm{KD}_{3}\left(\mathrm{SeO}_{3}\right)_{2}$ is observed by a method that utilizes laser light refraction at the ferroelastic domain structures. The high sensitivity of this method enables us to detect the refracted light even above the transition point $\left(T_{c}\right)$. The intensity level extends down three orders of magnitude smaller than that caused by the domains below $T_{c}$. This implies the embryos of the ferroelastic domains already exist in the high-temperature phase as the inhomogeneous strain field.

Observation of the structures and dynamics of domain formation in crystals is one of the central problems of nonequilibrium pattern formation both from technologi$\mathrm{cal}^{1}$ and scientific points of view. The domain structures are often investigated by means of optical microscopy, ${ }^{2}$ electron microscopy, ${ }^{3}$ force microscopy, ${ }^{4}$ light scattering, ${ }^{5}$ and so on. Among these techniques, the light deflection and diffraction from the domains has appeared as a powerful tool to observe domains. This method utilizes the difference of the optical indicatrix between adja- cent domains. $^{6}$ In site of its excellent sensitivity, there has been no report of the application of this method to investigate the initial process of domain formation. In this paper, we first apply this method to observe the domain formation just above the transition point and to show that even an extremely early stage of the equilibrium domain formation is observable in the ferroelastic phase transition.

In Fig. 1, we show the image of light scattered by a crystal of $\mathrm{KD}_{3}\left(\mathrm{SeO}_{3}\right)_{2}$ (DKTS) under Ar-laser illumina-

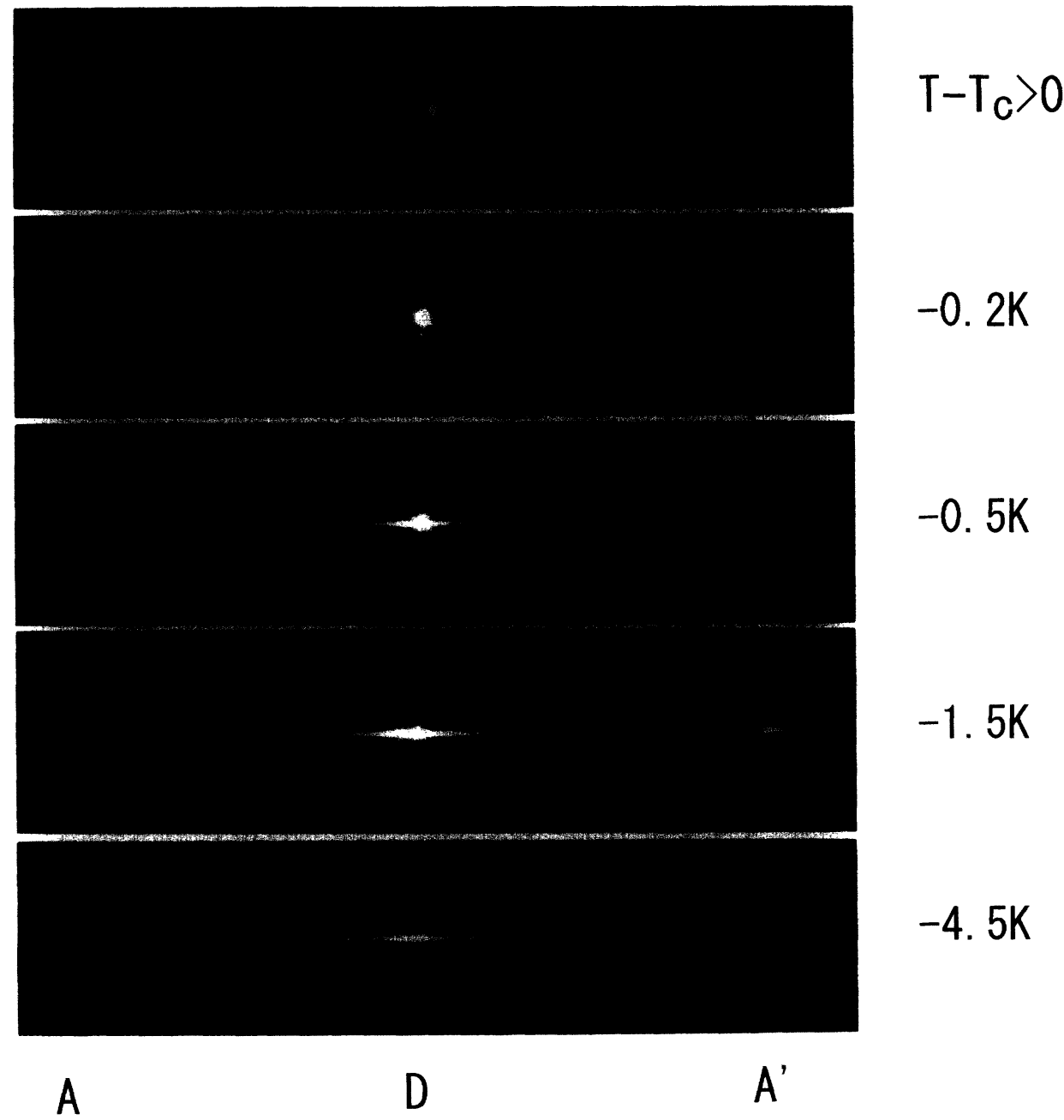

FIG. 1. Temperature dependence of the intensity distribution of transmitted and refracted light spots on the screen. The laser light was incident perpendicularly on the $b c$ plane of $\mathrm{KD}_{3}\left(\mathrm{SeO}_{3}\right)_{2}$ crystal. 
tion. This crystal undergoes a ferroelastic phase transition at $\sim 27^{\circ} \mathrm{C}$, accompanied by a softening of the shear strain $s_{4}{ }^{7}$ The crystal system changes its symmetry from orthorhombic $\left(P b c n-D_{2 h}^{14}\right)$ to monoclinic $\left(P 2_{1} / b-C_{2 h}^{5}\right)$ with decreasing temperature. From measurements of heat capacity, ${ }^{8}$ light scattering, ${ }^{9}$ and neutron diffraction, ${ }^{10}$ the type of this phase transition is concluded to be of second order. At the low-temperature phase, a stripe-shaped domain structure appears. The domain wall runs along the $a b$ or $a c$ plane. ${ }^{7}$ The photographs of Fig. 1 were obtained under the condition that the laser light was incident perpendicular to the $b c$ plane (cleavage plane) with the polarization direction perpendicular to the domain wall. When the temperature of the crystal is much higher than the transition point $\left(T_{c}\right)$, only the directly propagating light is observed (indicated as $D$ ). When the temperature is lowered below $T_{c}$, spots appear on both sides of $D$. The intensities of the two spots $A$ and $A^{\prime}$ increase with decreasing temperature. Finally all the light spots $A, A^{\prime}$, and $D$ spread as shown in Fig. 1 . Considering the relation between the incident and deflected angles of the light, we assigned these two spots to light refracted at a domain wall. ${ }^{11}$

We measured the temperature dependence of the intensity of the light spot $A$ or $A^{\prime}$ in detail near $T_{c}$. For this purpose, a beam from the Ar laser of $488 \mathrm{~nm}$ was attenuated sufficiently to avoid any local heating of the crystal. The refracted beam was collected through an aperture and detected by a photomultiplier. To reduce the scattering at the surface of the crystal, an appropriate refractive index matching was performed. The crystal was supported in a small copper cell which was temperature controlled within $\pm 5 \mathrm{mK}$. A newly cleaved surface was employed for the experiment. One-day thermal annealing at $50^{\circ} \mathrm{C}$ and repeated "temperature cycling" across $T_{c}$ were found necessary to reduce residual defects. After these procedures, the intensity of the refracted light appeared quite reproducible. The result is shown in Fig. 2. With decreasing temperature, the intensity of the refracted light increases nearly linearly in the region just below $T_{c}$, while it is almost saturated in the region

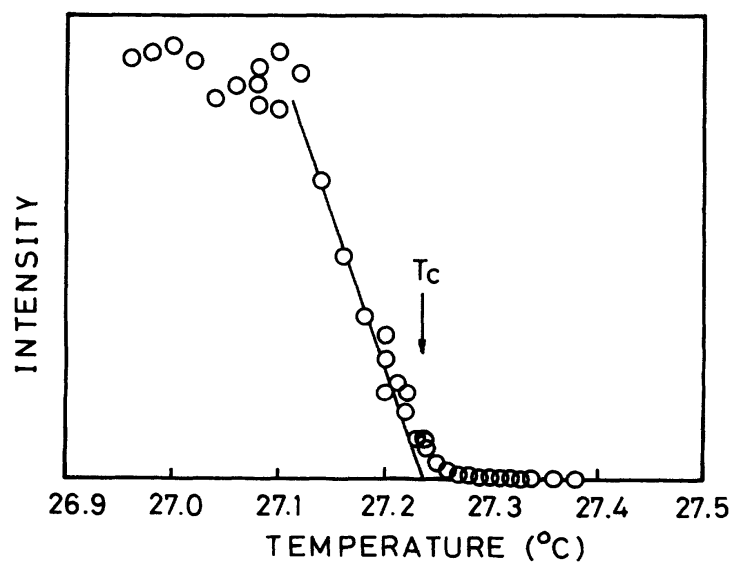

FIG. 2. Temperature dependence of the refracted light intensity from $\mathrm{KD}_{3}\left(\mathrm{SeO}_{3}\right)_{2}$ crystal. much below $T_{c}$. We have defined $T_{c}$ by extrapolation of the straight line to zero intensity in Fig. 2 . It is noticeable that a slight deviation from the straight line is observed around $T_{c}$. We have investigated this region in detail by operating the photomultiplier with high sensitivity, and have found that the highly directional light is observed even above $T_{c}$. The intensity of the observed light is more than two orders of magnitude higher than that of the stray light even well above $T_{c}$. The result is shown in Fig. 3, in which the intensities of the refracted light are plotted on a logarithmic scale. The light intensity decreases quickly at first and then gently with rising temperature. It is rather surprising that the light observed above $T_{c}$ originates from refraction at the domain wall, because the domain structures are known to form well below $T_{c}$. This phenomenon should be considered a new type of pretransitional behavior of ferroelastic phase transition. In the following, we investigate the origin of this deflected light and will show that it is related to an extremely early stage of the domain formation process.

Refraction of light at a domain wall originates from the fact that the principal axes of the optical indicatrix are different between adjacent domains. In DKTS, the directions of the principal axes are determined from the following dielectric tensor at optical frequencies. Namely, the dielectric tensor changes its form from orthorhombic to monoclinic as

$$
\left(\begin{array}{ccc}
\varepsilon_{11} & 0 & 0 \\
0 & \varepsilon_{22} & 0 \\
0 & 0 & \varepsilon_{33}
\end{array}\right) \rightarrow\left(\begin{array}{ccc}
\varepsilon_{11} & 0 & 0 \\
0 & \varepsilon_{22} & \varepsilon_{23} \\
0 & \varepsilon_{23} & \varepsilon_{33}
\end{array}\right),
$$

where the off-diagonal element $\varepsilon_{23}$ appearing in the monoclinic phase is connected with a mean spontaneous strain $\left\langle s_{4}\right\rangle$ in a domain as $\varepsilon_{23}=-\varepsilon_{0}^{2} p_{44}\left\langle s_{4}\right\rangle$. Here, $p_{44}$ is an element of the elasto-optic tensor of fourth rank, ${ }^{12} \varepsilon_{0}$ is the permittivity of vacuum, and $\left\langle s_{4}\right\rangle$ indicates the thermal average of $s_{4}$ which takes nonzero value below

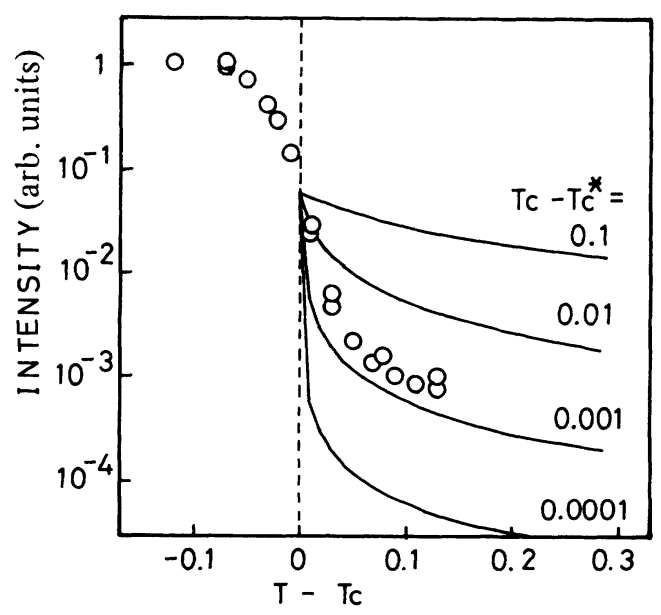

FIG. 3. Logarithmic plot of the refracted light intensity from $\mathrm{KD}_{3}\left(\mathrm{SeO}_{3}\right)_{2}$ crystal around the ferroelastic phase transition point. The simulations using Eq. (6) for various values of $T_{c}-T_{c}^{*}$ are shown as solid curves. 
$T_{c}$ corresponding to the spontaneous strain. The principal axes of the optical indicatrix are determined by diagonalizing the dielectric tensor in the monoclinic phase. The angle $\theta$ between the axis 2 or 3 in the orthorhombic phase and the principal axis of the monoclincic phase is expressed approximately as $\tan \theta \sim-\varepsilon_{0}^{2} p_{44}\left\langle s_{4}\right\rangle /$ $\left(\varepsilon_{33}-\varepsilon_{22}\right)$. Hence the inclination of the principal axis $\theta$ and the mean spontaneous strain $\left\langle s_{4}\right\rangle$ are proportional to each other for small $\theta$.

Consider the case where light propagates within a domain approximately parallel to the domain wall along the $a$ axis with the polarization direction coincident with one principal axis of the optical indicatrix. Then the direction of the electric displacement vector of the light differs by $2 \theta$ from that in the neighboring domain. Therefore, when the light hits the domain wall, the difference between the directions of the principal axes causes the refraction and reflection at the domain wall. Then, light having a polarization perpendicular to the incident light is generated. This corresponds to the refracted light, as far as the refractive index for the generated component is large compared with the original one. The intensity of the refracted light at the wall is thus expressed as $I_{r} \propto \sin ^{2} 2 \theta$. In this discussion, the inclination angle $\theta$ is again assumed sufficiently small, so that multiple refraction-reflection at subsequent walls can be neglected. This assumption is valid near $T_{c}$, because of the second-order nature of the phase transition.

When the phase transition is of second order, Landau theory predicts $\left\langle s_{4}\right\rangle \propto\left(T_{c}-T\right)^{1 / 2}$ for $T<T_{c}$, where $T$ is the temperature of the sample. Then, the intensity of the refracted light at a domain wall should be expressed as $I_{r} \propto T_{c}-T$. In fact, as shown in Fig. 2, the temperature dependence of the refracted light intensity changes almost linearly with $T_{c}-T$ below $T_{c}$. The saturation of the intensity at much lower temperatures is closely related to the spatial spread of the light spots observed in Fig. 1 and is explained by multiple refraction-reflection at many domain walls. The detailed discussion of this phenomenon will appear in the near future.

The above critical behavior of the refracted light intensity may be explained in terms of the critical fluctuation of the strain related to the domain formation. In order to understand this phenomenon qualitatively, we consider here the light refracted from the spatially distributed strains in crystal. Namely, since the refracted light originates from spatial variations of the optical indicatrix, light with different polarization from that of the incident light appears, whether the direction of the principal axis of the ellipsoid varies smoothly or abruptly. To consider these two cases consistently, we present here a continuum model of light refraction near the transition point.

Consider a stripe-shaped domain as in DKTS. We take the $z$ axis as perpendicular to the domain wall, with the wave vectors of the incident and refracted light as $\mathbf{k}_{0}$ and $\mathbf{k}_{1}$, respectively. For simplicity, we further neglect the light refraction-reflection at the crystal surface and also the difference of the refractive indices between the crystal and the surrounding space. The amplitude of electric field, which is caused by the spatial distribution of the strain, is expressed as

$$
E_{r} \propto \int_{V} d \mathbf{r}[d \theta(z) / d z] \exp \left\{i\left(\mathbf{k}_{0}-\mathbf{k}_{1}\right) \cdot \mathbf{r}\right\},
$$

at a sufficient distance from the crystal $\left(\int_{V} d \mathbf{r}\right.$ indicates that the integral is performed over the whole volume of the crystal). The intensity of the refracted light is then expressed as

$$
\begin{aligned}
I_{r} \propto \int d z^{\prime} \int & d z^{\prime \prime} \exp \left\{i\left(k_{0 z}-k_{1 z}\right)\left(z^{\prime}-z^{\prime \prime}\right)\right\} \\
& \times\left\langle\frac{d \theta\left(z^{\prime}\right)}{d z^{\prime}} \frac{d \theta\left(z^{\prime \prime}\right)}{d z^{\prime \prime}}\right\rangle \\
& \times \delta\left(k_{0 x}-k_{1 x}\right) \delta\left(k_{0 y}-k_{1 y}\right),
\end{aligned}
$$

where the correlation function with respect to the derivative of $\theta$ is assumed to only be dependent on the difference $\left|z^{\prime}-z^{\prime \prime}\right|$ and $\langle\cdots\rangle$ indicates the average over the distribution of the strain.

We first evaluate Eq. (1) within a framework of meanfield theory. Namely, the free energy of this system above $T_{c}$ is considered to be expressed as

$$
F=\int_{V} d \mathbf{r}\left[\frac{1}{2} \tau \psi^{2}+\frac{1}{2} C(\nabla \psi)^{2}\right],
$$

where $\tau \equiv A\left(T-T_{c}\right) . A(>0)$ and $C(>0)$ are constants independent of the temperature and $\psi \equiv \psi(T, z)$ is an order parameter. Since the structure factor obtained for the above free energy shows an Ornstein-Zernike-type behavior, the spatial correlation function of the order parameter is obtained as

$$
\langle\psi(z) \psi(0)\rangle=(1 / \sqrt{C \tau}) \exp (-|z| / \xi),
$$

where $\xi\left(\equiv(C / \tau)^{1 / 2}\right)$ indicates the correlation length. Inserting Eq. (3) into Eq. (1), we obtain

$$
\begin{aligned}
I_{r} \propto & \left(\tau / C^{2}\right)\left\{\left(k_{0 z}-k_{1 z}\right)^{2}+\xi^{-2}\right\}^{-1} \\
& \times \delta\left(k_{0 x}-k_{1 x}\right) \delta\left(k_{0 y}-k_{1 y}\right) .
\end{aligned}
$$

Equation (4) indicates that the intensity of the refracted light is rather constant with temperature, whenever the correlation length $\xi$ is shorter than the inverse of the wave-number difference of the lights. On the other hand, the light intensity decreases remarkably with $T-T_{c}$, when $\xi$ becomes larger than $\left|k_{0 z}-k_{1 z}\right|^{-1}$. This is because the intensity of the refracted light is connected with the derivative of the spatial variation of the strain. Namely, by increasing the correlation length, the spatial variation becomes so smooth that no refraction occurs. This result conflicts with the experimental result, in which an increase in the intensity is observed when the temperature comes close to $T_{c}$. This indicates that the critical fluctuation of the strain which takes place uniformly over the whole crystal is essentially irrelevant to the observed light refraction near $T_{c}$.

An alternative explanation of the experimental result is that in the high-temperature phase near the transition point, the crystal is assumed not to be completely uniform and background structure associated with the domain structure below $T_{c}$ is considered to exist in advance. The presence of this background structure in fer- 
roelastic crystals seems to be inevitable. It was already noticed by one of the present authors as a central peak in the light scattering spectra. ${ }^{13}$ Though the origin of these structures is not yet known, defects or dislocations of the crystal are the most probable causes.

The order parameter $\psi(T, z)$ in this case is expressed as the product of temperature-dependent and independent terms as

$$
\psi(T, z)=\chi(T) R(z),
$$

where $R(z)$ expresses a random function reflecting the background structure within the crystal and $\chi(T)$ is the temperature-dependent amplitude of the strain fluctuation. In the above expression, we further assume that the correlation length of the background structure is not so long that the strain fluctuates uniformly within this correlation length near $T_{c}$. Inserting Eq. (5) into Eq. (2), we obtain the free energy in this case:

$$
\begin{aligned}
F & =\chi^{2}(T) \int_{V} d \mathbf{r}\left\{\frac{1}{2} \tau R^{2}+\frac{1}{2} C[d R(z) / d z]^{2}\right\}, \\
& \equiv \frac{1}{2} \tau^{\prime} \chi^{2}(T),
\end{aligned}
$$

where $\tau^{\prime}$ is expressed as $\tau^{\prime} \equiv A^{\prime}\left(T-T_{c}^{*}\right)$ with

$$
T_{c}^{*} \equiv T_{c}-C \int_{V}(d R / d z)^{2} d \mathbf{r} /\left\{A \int_{V} R^{2} d \mathbf{r}\right\}
$$

and

$$
A^{\prime} \equiv A \int_{V} R^{2} d \mathbf{r}
$$

The intensity of the refracted light is then expressed as

$$
\begin{aligned}
I_{r} \propto & \left\langle\chi^{2}\right\rangle\left\langle\left|\bar{R}\left(k_{0 z}-k_{1 z}\right)\right|^{2}\right\rangle \\
& \times \delta\left(k_{0 x}-k_{1 x}\right) \delta\left(k_{0 y}-k_{1 y}\right) \\
\propto & \tau^{\prime-1}=\left\{A^{\prime}\left(T-T_{c}^{*}\right)\right\}^{-1},
\end{aligned}
$$

where $\bar{R}^{\prime}(k)$ is a Fourier component of $(d R / d z)$ with the period of $k$. We have simulated the intensity of the re- fracted light for various values of $T_{c}-T_{c}^{*}$. The results of this calculation are shown in Fig. 3. They reproduce the temperature dependence of the intensity above $T_{c}$ well. Good agreement between experiment and calculation is obtained when $T_{c}-T_{c}^{*} \sim 0.001 \mathrm{~K}$, suggesting the effectiveness of the present consideration for $I_{\mathrm{r}}$ indicated by Eq. (6).

The present result seems consistent with the previous spectroscopic study which reported a central-peak spectrum of ferroelastic $\mathrm{KH}_{3}\left(\mathrm{SeO}_{3}\right)_{2}$ and $\mathrm{KD}_{3}\left(\mathrm{SeO}_{3}\right)_{2}$ crystals. ${ }^{13}$ The physical origin of the central peak has been assigned to the inhomogeneous strain field conjugate to the shear strain $s_{4}$. The strain field denoted by $R(z)$ in Eq. (5) corresponds to the origin of the central peak phenomena. In addition, deuterated $\mathrm{KD}_{3}\left(\mathrm{SeO}_{3}\right)_{2}$ crystals contain many static lattice defects at the deuteron sites caused by the isotopic abundance of $\mathrm{H}$ atoms among $\mathrm{D}$ atoms. These defects might be responsible for the static distribution $R(z)$ of strains in the extremely early stage above $T_{c}$. The physical origin of $T_{c}^{*}$ is not clear at the present stage. However, as shown in the definition, it is very closely related with the domain structure near the transition point. Hence, the precursor of the domain appeared as the inhomogeneous strain field seems to disturb the uniform critical fluctuation and may slightly depress the transition point.

In summary, we have observed the light refracted at the domain wall beyond $T_{c}$, and have shown that an extremely early stage of the domain formation process is detectable as a new type of pretransitional behavior. This method of observing the refracted light intensity is suitable to investigate the early stage of the critical behavior in ferroelastic crystal. Well above $T_{c}$, detection in the direction of the refracted light is free from stray light under proper index matching and hence extremely weak light is observable. The application of this method to various types of domains is promising, because most ferromagnetic and ferroelectric crystals accompany the generation of the spontaneous strain with phase transitions. Much effort along this course is clearly needed.
*Present address: Department of Physics, Faculty of Science, Osaka University, Toyonaka, Osaka 560, Japan.

1S. W. Meeks, Adv. Electron. Electron. Phys. 71, 251 (1988).

${ }^{2}$ M. E. Lines and A. M. Glass, Principles and Applications of Ferroelectrics and Related Materials (Clarendon, Oxford, 1979), p. 87.

${ }^{3}$ R. Le Bihan and M. Maussion, J. Phys. (Paris) Colloq. 33, C2215 (1972)

${ }^{4}$ F. Saurenback and B. D. Terris, Appl. Phys. Lett. 56, 1703 (1990).

${ }^{5}$ S. M. Shapiro, R. W. Gammon, and H. Z. Cummins, Appi. Phys. Lett. 10, 113 (1967); M. Nakamura, H. Orihara, Y. Ishibashi, and K. Hara, J. Phys. Soc. Jpn. 59, 4472 (1990).

${ }^{6}$ R. M. Hill and S. K. Ichiki, Phys. Rev. 135, A1640 (1964); T. Tsukamoto, J. Hatano, and H. Futama, J. Phys. Soc. Jpn. 51, 3948 (1982).

${ }^{7}$ N. R. Ivanov, L. A. Shuvalov, and N. V. Gordeeva, Sov. Phys.
Crystallogr. 13, 145 (1968); T. Yagi and Z. Lu, in Dynamics of Ordering Processes in Condensed Matter, edited by S. Komura and H. Furukawa (Plenum, New York, 1988), p. 343.

${ }^{8}$ Y. Makita, Y. Yamachi, and S. Suzuki, J. Phys. Soc. Jpn. 43, 181 (1977)

${ }^{9}$ H. Tanaka, T. Yagi, and I. Tatsuzaki, J. Phys. Soc. Jpn. 44, 1257 (1978); M. Copic, M. Zgonik, D. L. Fox, and B. B. Lavrencic, Phys. Rev. B 23, 3469 (1981).

${ }^{10}$ Y. Noda, R. Youngblood, G. Shirane, and Y. Yamada, J. Phys. Soc. Jpn. 48, 1576 (1980).

${ }^{11}$ T. Tsukamoto, T. Sato, J. Hatano, N. Tamano, and H. Futama, Jpn. J. Appl. Phys. Suppl. 24-2, 559 (1985).

12J. F. Nye, Physical Properties of Crystals (Clarendon, Oxford, 1972), p. 243.

${ }^{13}$ T. Yagi, H. Tanaka, and I. Tatsuzaki, Phys. Rev. Lett. 38, 609 (1977). 


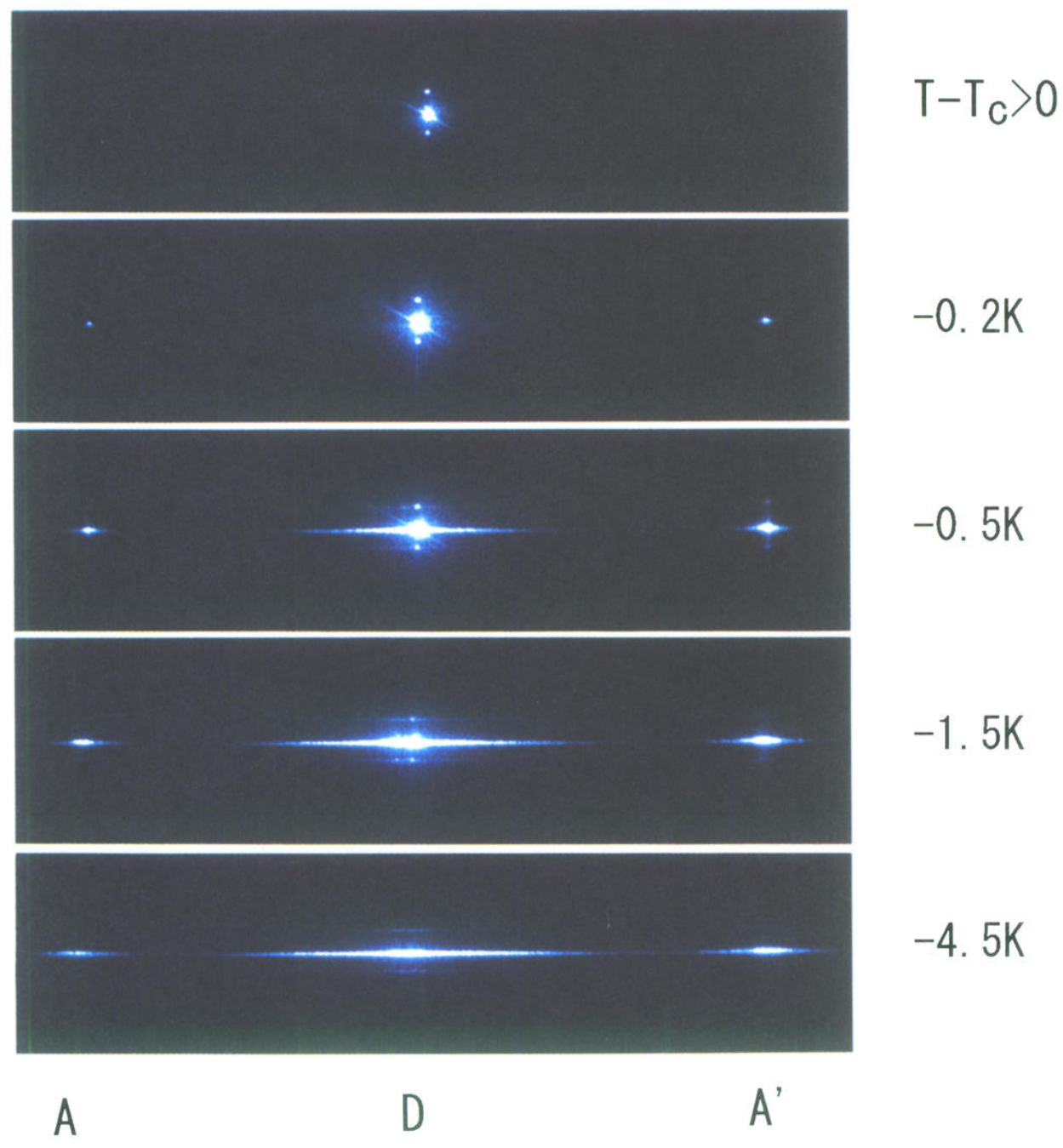

FIG. 1. Temperature dependence of the intensity distribution of transmitted and refracted light spots on the screen. The laser light was incident perpendicularly on the $b c$ plane of $\mathrm{KD}_{3}\left(\mathrm{SeO}_{3}\right)_{2}$ crystal.
A
D

A 